\title{
Effect of Rab23 on the proliferation and apoptosis in breast cancer
}

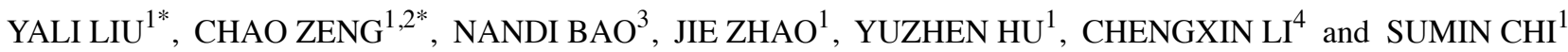 \\ ${ }^{1}$ Department of Physiology, State Key Discipline of Cell Biology, The Fourth Military Medical University, Xi'an, \\ Shaanxi 710032; ${ }^{2}$ Department of Cardiology, No. 421 Hospital of PLA, Guangzhou, Guangdong 510318; \\ ${ }^{3}$ Team 2, Cadet Brigade, School of Stomatology and ${ }^{4}$ Department of Dermatology, \\ Chinese People's Liberation Army General Hospital, Beijing 100853, P.R. China
}

Received April 8, 2015; Accepted June 26, 2015

DOI: 10.3892/or.2015.4152

\begin{abstract}
Rab23, as a negative regulatory molecule of the Hedgehog (Hh) signaling pathway, may be a new target for treating carcinoma. In the present study, we aimed to determine whether Rab23 is expressed in breast cancer cells and whether Rab23 affects the viability and proliferation of breast cancer cells. We evaluated Rab23 expression in several breast cancer cell lines including MDA-MB-231, Bcap37 and MCF-7 by reverse transcription-PCR (RT-PCR), western blotting and immunofluorescence in vitro. We assessed cell growth and proliferation by 3-(4,5-dimethylthiazol-2-y1)-3,5-diphenyltetrazolium bromide (MTT), colony formation and bromodeoxyuridine (BrdU) incorporation assays. The distribution of the cell cycle and the rate of apoptosis were assessed using flow cytometry (FCM). In addition, we determined the mechanisms by which Rab23 regulates the Hh pathway by detecting the level of Gli molecules by RT-PCR. We found that Rab23 mRNA and protein levels were expressed in breast cancer cells, and the expression of Rab23 in MDA-MB-231 cells was higher than that in the MCF-7 cells. Rab23 protein was primarily expressed and localized in the cytoplasm surrounding the nucleus. The MTT assay showed that the absorbance value at $\mathrm{A}_{490 \mathrm{~nm}}$ of the Rab23-transfected group was reduced in comparison with the control group. The number of colonies formed in the breast cancer cells was significantly reduced and BrdU labeling was weakened in the
\end{abstract}

Correspondence to: Professor Sumin Chi, Department of Physiology, The Fourth Military Medical University, 17 Changle West Road, Xi'an, Shaanxi 710032, P.R. China

E-mail: chism1201@163.com

Dr Chengxin Li, Department of Dermatology, Chinese People's Liberation Army General Hospital, 28 Fuxing Road, Beijing 100853, P.R. China

E-mail: chengxinderm@163.com

${ }^{*}$ Contributed equally

Key words: Rab23, hedgehog signaling pathway, breast cancer, proliferation, apoptosis group transfected with Rab23. The results of FCM showed that overexpression of Rab23 protein caused cell cycle arrest in the G1 phase and a decrease in the $\mathrm{S}$ phase population as well as induction of apoptosis. Furthermore, Rab23 decreased Gli1 and Gli2 mRNA levels when compared with the control group. Our results indicate that Rab23 is expressed in breast cancer cells, and ectopic expression of Rab23 inhibits the growth and proliferation as well as induces cell apoptosis in breast cancer cells. These effects may be due to the inhibition by Rab23 of Gli1 and Gli2 mRNA expression. These results suggest that Rab23 is a potential target for the treatment of breast cancer.

\section{Introduction}

Breast cancer, the second leading cause of cancer-related mortality among women, has become an increasingly important public health issue worldwide. It threatens the health of women all over the world (1). For example, 230,480 new cases of invasive breast cancer and 39,520 breast cancer deaths were estimated to occur among US women in 2011, accounting for nearly 1 in 3 cancers diagnosed among women in the US. The biological features of breast cancer are still not well understood, however, much has changed in the management of breast cancer particularly over the last few decades. Several effective treatments have been explored in dealing with different types of advanced breast cancer using newly developed targeted therapy, such as endocrine therapy and trastuzumab treatment in HER-2 $2^{+}$metastatic breast cancer. Therefore, together, oncologists and clinicians are working diligently to discover more target molecules of breast cancer and are aimed at developing more individual-based strategies for breast cancer patients. Consequently, there is a growing list of such targeting molecules in breast cancers. The Hedgehog (Hh) signaling pathway has recently been recognized as one of the most important signaling pathways and a therapeutic target in cancer.

The Hh signaling pathway has three ligands: sonic $(\mathrm{SHH})$, Indian (IHH) and desert (DHH) hedgehogs. When the ligands bind to their Patched (PTC), a 12-transmembrane receptor, the inhibition of PTC on Smoothened (SMO), a 7-transmembrane protein, is removed $(2,3)$. Therefore, the signaling pathway is active, resulting in the activation of the zinc finger transcrip- 
tion factors Gli1, Gli2 and Gli3, where Gli1 and Gli2 represent the main activators of Hh target genes and Gli3 acts mostly as a repressor. The exact mechanism of signal transduction from Smo to the Gli proteins is not yet clear; however, it has been proposed that regulating Gli transcription factors involves a multi-protein complex consisting of intraflagellar transport proteins, protein kinase A, casein kinase, glycogen synthase kinase 3 , suppressor of fused and others. Constitutive activation of the Hh pathway leading to tumorigenesis has been noted in basal cell carcinomas, medulloblastoma, gastrointestinal tumors and lung and prostate cancers (4). Breast cancer is also associated with the aberrant activation of the $\mathrm{Hh}$ signaling pathway (5). A high-resolution comparative genomic hybridization analysis of breast cancer samples revealed a frequent loss of PTCH1 (9q22.1-q31) and amplification of Gli1 (12q13.2-q13.3) chromosomal regions, indicating that Hh signaling is involved in breast cancer $(6,7)$. Evidence has shown that expression of SHH, DHH and Gli1 is higher in various breast cancer cell lines and in $30 \%$ of cancer samples compared with normal mammary epithelial cells or epithelial tissue $(8,9)$. More importantly, active human SMO (SmoM2) in the mammary epithelium as well as overexpression of Gli2 both resulted in ductal hyperplasia (10). Furthermore, a genome-wide RNAi screening demonstrated that Gli2 is necessary for the growth of breast cancer cell lines and cyclopamine treatment could induce apoptosis of breast cancer cell lines with elevated SHH, DHH and Gli1 protein expression $(8,9,11)$. Although, the data postulating the involvement of this pathway in human breast cancer are still limited, increasing evidence implies that the Hh signaling pathway is potentially a useful target in multi-targeted breast cancer therapy. Modulating its activity may affect cancer progression and metastasis.

Rab23 is also known to play a key role in negatively regulating $\mathrm{Hh} / \mathrm{Gli}$ signaling, since targeted disruption of the murine Rab23 gene leads to neural tube defects, resembling the phenotype caused by an excess of Hh signaling, and mutation of the Rab23 gene causes abnormalities of multiple organs in patients of two congenital diseases - Carpenter and Gorlin syndromes. Since over-activation of the Hh signaling pathway is oncogeneic in human cancers, Rab23, a negative regulator of the Hh signaling pathway, has been postulated to be a suppressor gene in carcinogenesis. The abnormal expression of Rab23 has been observed in hepatocellular as well as thyroid carcinoma (12). The overexpression of Rab23 has been reported in atrophic gastritis, a pre-stage of gastric cancer (13). Atrophic gastritis may increase the risk of gastric cancer, and these findings raise the possibility that Rab23 upregulation may be involved at an early stage of gastric carcinogenesis.

To date there have been no studies concerning Rab23 expression in breast cancer. Since Rab23 is a negative regulator of Hh signaling which can induce malignant carcinoma, upregulation of Rab23 may be helpful in the treatment of breast cancer. If this is true, we may be able to identify a new target for the diagnosis and treatment of breast cancer. In the present study, we demonstrated that Rab23 was expressed in MCF-7 and MDA-MB-231 cell lines, and Rab23 activity inhibited breast cancer cell proliferation and DNA synthesis, and induced apoptosis and downregulated Gli1 and Gli2 expression, which is similar to the effects of cyclopamine on breast cancer cells in vitro.

\section{Materials and methods}

Cell culture. Breast cancer cell lines, MDA-MB-231, MCF-7 and Bcap37, were obtained from the Cell Resource Center of Shanghai Institute for Biological Sciences (Chinese Academy of Sciences, Shanghai, China). They were maintained in RPMI-1640, supplemented with $10 \%$ heat-inactivated $\left(56^{\circ} \mathrm{C}\right.$ for $30 \mathrm{~min}$ ) newborn calf serum (both from Hyclone, Logan, UT, USA) and antibiotics (100 U/ml penicillin and $100 \mu \mathrm{g} / \mathrm{ml}$ streptomycin). Cells were cultured at $37^{\circ} \mathrm{C}$ in a humidified incubator containing $95 \%$ air and $5 \% \mathrm{CO}_{2}$. Cells were seeded into 96-, 24- and 6-well plates or a culture bottle, and serum-starved $24 \mathrm{~h}$ before cell transfection.

Immunofluorescence analysis. Cells $\left(2 \times 10^{5} /\right.$ well) were incubated on 24-well plates (Corning Costar, Cambridge, MA, USA) at $37^{\circ} \mathrm{C}$ which were covered with coverslips $(1 \mathrm{x} 1 \mathrm{~cm})$. When the cells grew to occupy $\sim 80 \%$ of the coverslips, the cells were fixed in $4 \%$ paraformaldehyde for $30 \mathrm{~min}$ at room temperature. After that, endogenous peroxidase activity was blocked by using $3 \% \mathrm{H}_{2} \mathrm{O}_{2}$ in methanol for $30 \mathrm{~min}$ at room temperature. After blocking with 5\% normal goat serum containing 3\% BSA in phosphate-buffered saline (PBS) for $1 \mathrm{~h}$ at $37^{\circ} \mathrm{C}$, the cells were incubated with the primary antibody, rabbit anti-Rab23 (1:100, sc-20687; Santa Cruz Biotechnology, Santa Cruz, CA, USA), in a moist chamber at $4^{\circ} \mathrm{C}$ overnight. After washing three times with PBS, the coverslips were incubated with secondary antibodies (1:200, FITC-conjugated goat anti-rabbit antibodies; Sigma-Aldrich, St. Louis, MO, USA) for $1 \mathrm{~h}$ at $37^{\circ} \mathrm{C}$ in the dark. The coverslips were lightly counterstained with Hoechst (1:100; Sigma-Aldrich). Coverslips were read in a fluorescence microscope at $\mathrm{x} 400$ magnification (Nikon, Japan). As a control for specificity of the primary antibody, coverslips were incubated with normal goat serum instead of the specific antibody during the first incubation. In such control sections, coverslip immunofluorescence was not observed.

Semi-quantitative reverse transcription-PCR (RT-PCR). Total RNA was extracted using TRIzol reagent (Invitrogen, Carlsbad, CA, USA) according to the manufacturer's instructions. Complementary DNA (cDNA) was synthesized from $5 \mu \mathrm{g}$ of total RNA with random primers following the manufacturer's instructions (MBI Fermantas, Vilnius, Lithuania). Semi-quantitative reverse transcribed-PCR (RT-PCR) was performed in two steps. First, $5 \mu \mathrm{g}$ RNA extraction of total RNA was reverse transcribed with $1 \mu 1$ reverse transcriptase (AMV-RT), $2 \mu \mathrm{l}$ deoxynucleotide triphosphates (dNTPs), $0.5 \mu 1 \mathrm{RNase}$ inhibitor, $4 \mu 1 \mathrm{MgCl}_{2}, 2 \mu \mathrm{l}$ Rev-Trans, $1 \mu \mathrm{l}$ primer oligo(dT), and $4.5 \mu \mathrm{l}$ nuclease-free water for $1 \mathrm{~h}$ at $47^{\circ} \mathrm{C}$ according to the manufacturer's instructions (Promega, Madison, WI, USA). Second, PCR amplification was performed with $0.25 \mu 1$ AmpliTaq DNA polymerase (Takara, Japan), $1.0 \mu \mathrm{l}$ each primer, $3 \mu 1 \mathrm{MgCl}_{2}$ and $10 \mathrm{mM}$ dNTPs in a thermocycler (Bio-Rad, Hercules, CA, USA). Sequences of the primers used in this study are listed on Table I. Each RT-PCR product $(10 \mu \mathrm{l})$ was analyzed by electrophoresis through a $2 \%$ agarose gel. PCR amplification signals were quantified by densitometric scanning using Quantity One (Bio-Rad). For primers, see Table I. 
Table I. RT-PCR primers for analysis.

\begin{tabular}{lll}
\hline Gene name & \multicolumn{1}{c}{ Forward primer } & \multicolumn{1}{c}{ Reverse primer } \\
\hline Rab23 & AGG CAC TGG CAA AAA GGT TA & TA GAC CAC CTT CAG TGA GGC \\
Gli1 & CGGGGTCTCAAACTGCCCAGCTT & GGCTGGGTCACTGGCCCTC \\
Gli2 & CTAGCATCAGCGAGAACGTG & AAAGCCTAACTGGCATCCTC \\
GAPDH & ACC ACA GTC CAT GCC ATC AC & TCC ACC ACC CTG TTG CTG TA
\end{tabular}

Protein extraction and western blotting. The cells were washed with cold PBS and lysed by the addition of a lysis buffer containing $1 \%$ Nonidet P-40, $50 \mathrm{mM}$ Tris ( $\mathrm{pH} 7.5), 150 \mathrm{mM} \mathrm{NaCl}, 0.1 \%$ SDS and protease inhibitor cocktail (Boehringer Mannhein, Lewes, UK) for $20 \mathrm{~min}$ at $4^{\circ} \mathrm{C}$. Insoluble materials were removed by centrifugation at $15,000 \mathrm{rpm}$ for $15 \mathrm{~min}$ at $4^{\circ} \mathrm{C}$. The supernatant was saved and the protein concentration was determined using a Bio-Rad protein assay kit (Bio-Rad). Cell extracts (50 $\mu \mathrm{g} /$ lane) were separated via $10 \%$ gel electrophoresis and electroblotted onto PVDF membranes. Nonspecific binding sites were blocked by incubating the nitrocellulose sheets for $1 \mathrm{~h}$ in PBS containing $5 \%$ low-fat dry milk. Membranes were probed with the primary anti-Rab23 antibody (1:1,000; Cell Signaling Technology, Inc., Danvers, MA, USA) overnight at $4^{\circ} \mathrm{C}$, followed by the horseradish peroxidase-conjugated secondary antibody. Blots were developed using an enhanced chemiluminescence detection system (ECL; Amersham Pharmacia Biotech) according to the manufacturer's instructions.

Rab23 gene silencing. In order to knock down protein expression, we administered specific small interfering RNA (siRNA) against Rab23 with specific siRNA for green fluorescent protein (GFP) as the control. The following siRNA sequences were applied (Shanghai GenePharma Co., Ltd., Shanghai, China): siRNA sequence (siRNA duplex), 5'-CUA CAG AAC AUC AGU GAA ATT-3' (sense) and 5'-UUU CAC UGA UGU UCU GUA GTT-3' (antisense); sequence of the negative control siRNA, 5'-UUC UCC GAA CGU GUC ACG UTT-3' (sense) and 5'-ACG UGA CAC GUU CGG AGA ATT-3' (antisense). Expression levels were analyzed $48 \mathrm{~h}$ after transfection via RT-PCR and immunofluorescence.

siRNA and cDNA transfection. Human Rab23 and Gli1 expression vectors were kindly provided by Dr J. Xie (Wells Center for Pediatric Research, Department of Pediatrics, The Indiana University Simon Cancer Center, Indiana University, USA). Cells were seeded into a 24- and a 6-well plate at a density of $2.5 \times 10^{4}$ cells/well and incubated for $24 \mathrm{~h}$. Then the cells were transfected with either Rab23 and Gli1 cDNA or specific Rab23 siRNA by Lipofectamine ${ }^{\mathrm{TM}} 2000$ transfection reagent in Opti-MEM medium (both from Invitrogen) according to the manufacturer's instructions. All transfection experiments were carried out in MDA-MB-231 and MCF-7 cells, and repeated at least 3 times using 2 sets of plasmids prepared separately. After $48 \mathrm{~h}$ of transfection, relative cell proliferation, cell cycle distribution and apoptosis were determined by measuring absorbance using bromodeoxyuridine (BrdU) and flow cytometry (FCM) assays. Each assay was carried out in triplicate, and normalized relative to the control-transfected cells.

Incorporation efficiency of BrdU. Cells (1x10 $/$ well) were incubated on 24 -well plates with coverslips $(1 \times 1 \mathrm{~cm})$ at $37^{\circ} \mathrm{C}$, after transfection with Rab23 or siRNA. When the cells occupied $\sim 80 \%$ of the coverslips, the cells were incubated in RPMI-1640 medium supplemented with $10^{-5} \mu \mathrm{mol} / 1$ BrdU (Zhongshan Goldenbridge, Beijing, China) for $15 \mathrm{~min}$ at $37^{\circ} \mathrm{C}$ in a humidified incubator. The cells were fixed in $4 \%$ paraformaldehyde for $30 \mathrm{~min}$ at room temperature. After being blocked with 5\% normal goat serum containing 3\% BSA in PBS for $1 \mathrm{~h}$ at $37^{\circ} \mathrm{C}$, the primary antibody $\operatorname{BrdU}(1: 100$; Santa Cruz Biotechnology) in blocking solution was incubated with the cells at $4^{\circ} \mathrm{C}$ overnight. After washing with PBS, the coverslips were then incubated with the secondary antibody (1:200; FITC-conjugated goat anti-rabbit antibody) for $1 \mathrm{~h}$ at $37^{\circ} \mathrm{C}$ in the dark. The slides were lightly counterstained with Hoechst (1:100; Sigma-Aldrich). Detection of the protein was visualized using fluorescence microscopy (Nikon, Japan). We randomly chose 5 fields visualized in every coverslip under fluorescence microscopy. Incorporation efficiency of BrdU in the tumor cells $=$ (the number of BrdU-positive cells/the number of cells) x $100 \%$.

3-(4,5-Dimethylthiazol-2-y1)-2,5-diphenyltetrazolium bromide (MTT) assay. The cell viability was determined by MTT assay. After transfection with the plasmids of Rab23 or siRNA into the MCF-7 and MDA-MB-231 cells for $24 \mathrm{~h}$, the cells $\left(5 \times 10^{3} /\right.$ well) were digested and seeded in 96-well microtiter plates (Costar, Denmark). When cells were incubated for 1, 3 or 5 days, an aliquot $(50 \mu \mathrm{l})$ of MTT solution ( $5 \mathrm{mg} / \mathrm{ml}$ in PBS; Sigma-Aldrich) was added to each well, and the plates were incubated for an additional $4 \mathrm{~h}$ at $37^{\circ} \mathrm{C}$. MTT solution was aspirated off, and $150 \mu \mathrm{l}$ dimethyl sulfoxide (DMSO) was added to each well to dissolve the formazan crystals formed in the viable cells for $10 \mathrm{~min}$. The absorbance was read at $490 \mathrm{~nm}$ on a Dias automatic microwell plate reader with DMSO as the blank. Each time point was performed in 3 wells.

Colony formation assay. MDA-MB-231 and MCF-7 cells $\left(2 \times 10^{4} /\right.$ well) were incubated on 6 -well plates at $37^{\circ} \mathrm{C}$. After incubation for $24 \mathrm{~h}$, the cells were transfected with the plasmids of Rab23 or Gli1. Twenty-four hours later, the cells were screened by G418 (400 $\mu \mathrm{g} / \mathrm{ml}$; Zhongshan Goldenbridge) for 10 days. Hexamethylpararosaniline (1\% in PBS) was used to stain the colonies. The colonies with $>50$ cells were counted by inverted microscopy. Colony-forming efficiency of the 


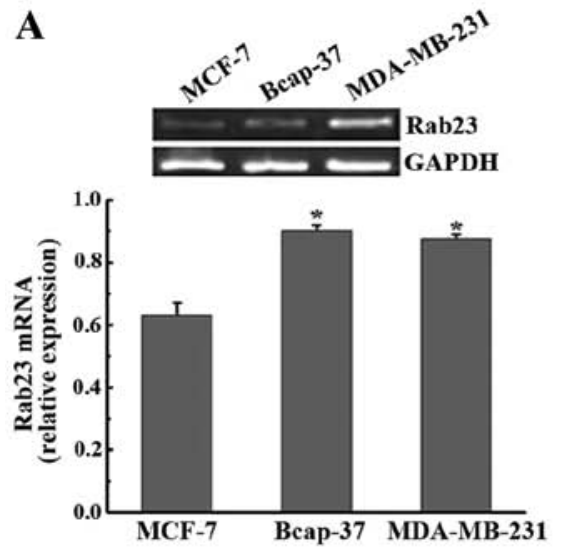

C
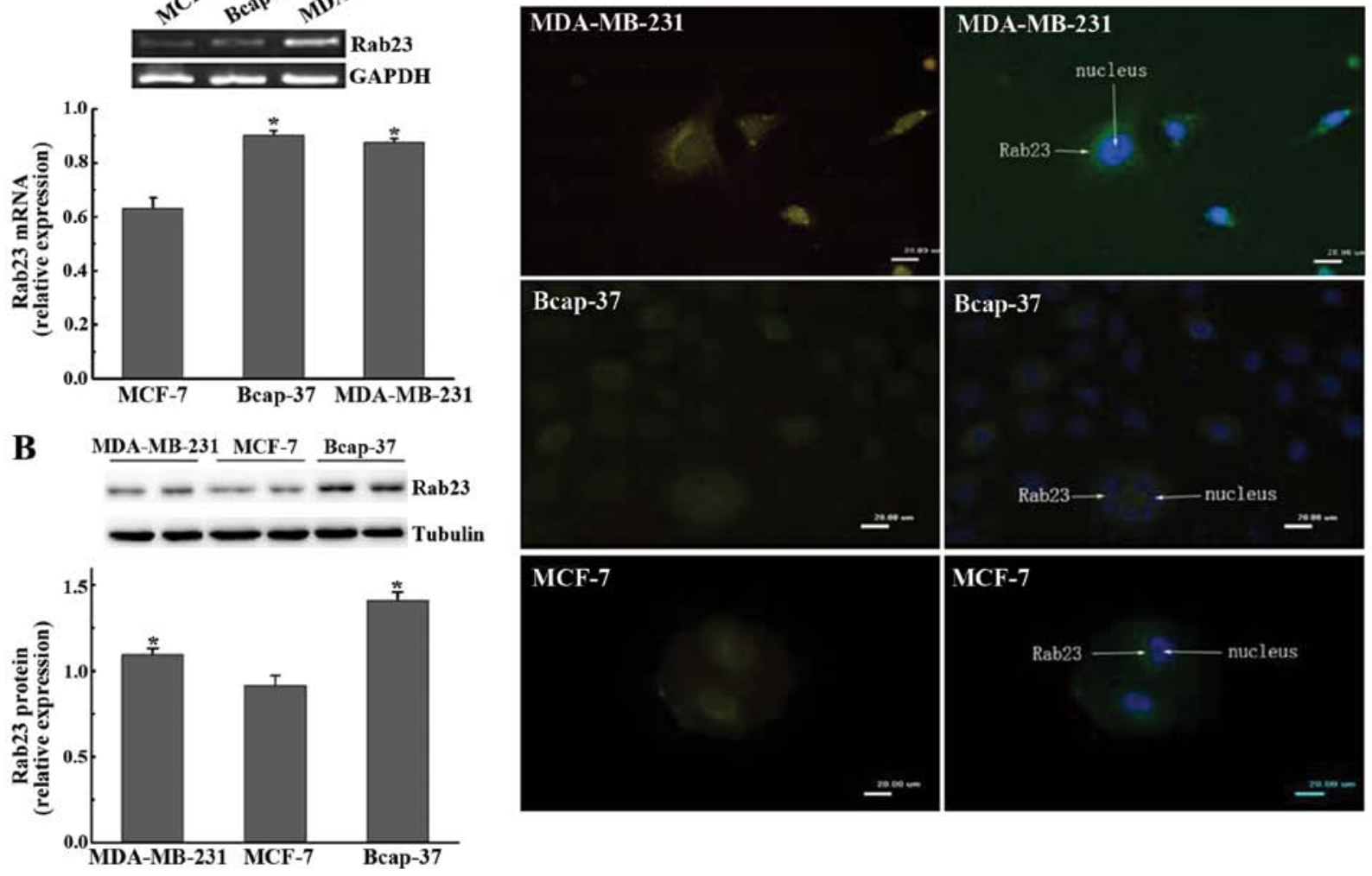

Figure 1. Expression of Rab23 in breast cancer cells. (A) Results of Rab23 expression as detected by RT-PCR. Rab23 mRNA was detected in breast cancer cell lines. The expression levels of Rab23 mRNA in the MDA-MB-231 and Bcap-37 cells were higher than that in the MCF-7 cells ("P<0.05), while there was no statistical difference observed between the MDA-MB-231 and Bcap-37 cells ( $\mathrm{P}>0.05)$. (B) Results of Rab23 protein expression as detected by western blotting. Rab23 protein was detected in the breast cancer cell lines. The expression of Rab23 protein in the MDA-MB-231 and Bcap-37 cells was higher than that in the MCF-7 cells. "P<0.05. (C) Validation of Rab23 expression by immunofluorescence. Rab23-FITC was detected by fluorescence microscope. After merging with cell nucleus labeled by Hoechst, we found that Rab23 protein was primarily expressed and localized in the cytoplasm in the MDA-MB-231 cells and around the cell nucleus in the Bcap-37 and MCF-7 cells. All the results are presented as the mean \pm SEM of four independent experiments. ${ }^{*} \mathrm{P}<0.05$.

tumor cells $=$ (the number of colonies/the number of seeded cells) x $100 \%$.

Cell cycle analysis by FCM. MCF-7 and MDA-MB-231 cells were seeded into 6-well plates and transfected either with Rab23 or siRNA as described above for $48 \mathrm{~h}$. Then the cells were harvested, washed in PBS, fixed with $70 \%$ ethanol at $4^{\circ} \mathrm{C}$ for $24 \mathrm{~h}$, followed by DNA staining with propidium iodide (PI, $1 \mu \mathrm{g} / \mathrm{ml}$; Sigma-Aldrich) to analyze cellular DNA content. Cell cycle analysis was performed on a flow cytometer (Beckman Coulter Inc., Miami, FL, USA).

Apoptosis assay. The apoptotic status of MCF-7 and MDA-MBA-231 cells was evaluated by measuring the exposure of phosphatidylserine on the cell membrane using Annexin V-fluorescein isothiocyanate (Annexin V-FITC) and PI staining by FCM. Breast cancer cells were plated in 6-well plates. When the cells occupied $\sim 70 \%$ of the wells, the cells were transfected with the plasmids of Rab23. After $24 \mathrm{~h}$ of incubation, the cells were digested and harvested. After centrifugation, the cell pellets were washed twice with PBS at $4^{\circ} \mathrm{C}$. The cells were incubated with $5 \mu \mathrm{l}$ Annexin V-FITC and $10 \mu \mathrm{l}$ PI at room temperature for $15 \mathrm{~min}$ in the dark. After the incubation, $250 \mu \mathrm{l}$ of $1 \mathrm{X}$ binding buffer was added to each tube. The cells were analyzed immediately by FCM (Beckman Coulter).
Statistical analysis. All values are expressed as the means \pm SEM. The significance of differences among groups was evaluated by a Student's t-test for unpaired data or Dunnett's t-test for multiple comparisons preceded by one-way analysis of variance (GraphPad Prism 5.0). For all tests, a P-value of $<0.05$ was considered to indicate statistical significance.

\section{Results}

Rab23 is expressed in the breast cancer cell lines. We selected breast cancer cell lines MDA-MB-23 Bcap37 and MCF-7, and determined whether Rab23 is expressed in these cell lines. We found that Rab23 mRNA and protein were expressed in all three cell lines. Expression of Rab23 mRNA and protein in the MDA-MB-231 and Bcap-37 cells was higher than that in the MCF-7 cells $(\mathrm{P}<0.05)$ (Fig. 1A and $\mathrm{B})$. From the immunofluorescence results, we found that Rab23 protein was expressed in the three breast cancer cell lines. However, its pattern of expression was different. Rab23 protein fluorescence was observed in the cytoplasm of the MDA-MB-231 cells but was observed around the cell nucleus in the Bcap-37 and MCF-7 cells (Fig. 1C). Since both Bcap37 and MDA-MB-231 cells are ER-negative, and the expression pattern had no significant difference, we chose MDA-MB-231 cells to perform the subsequent experiments. 


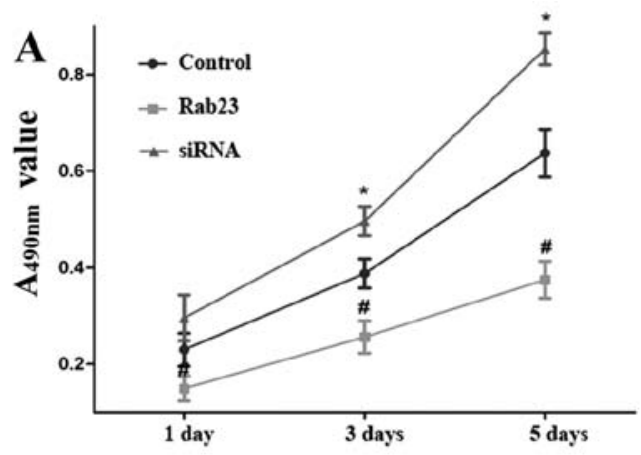

\section{C}
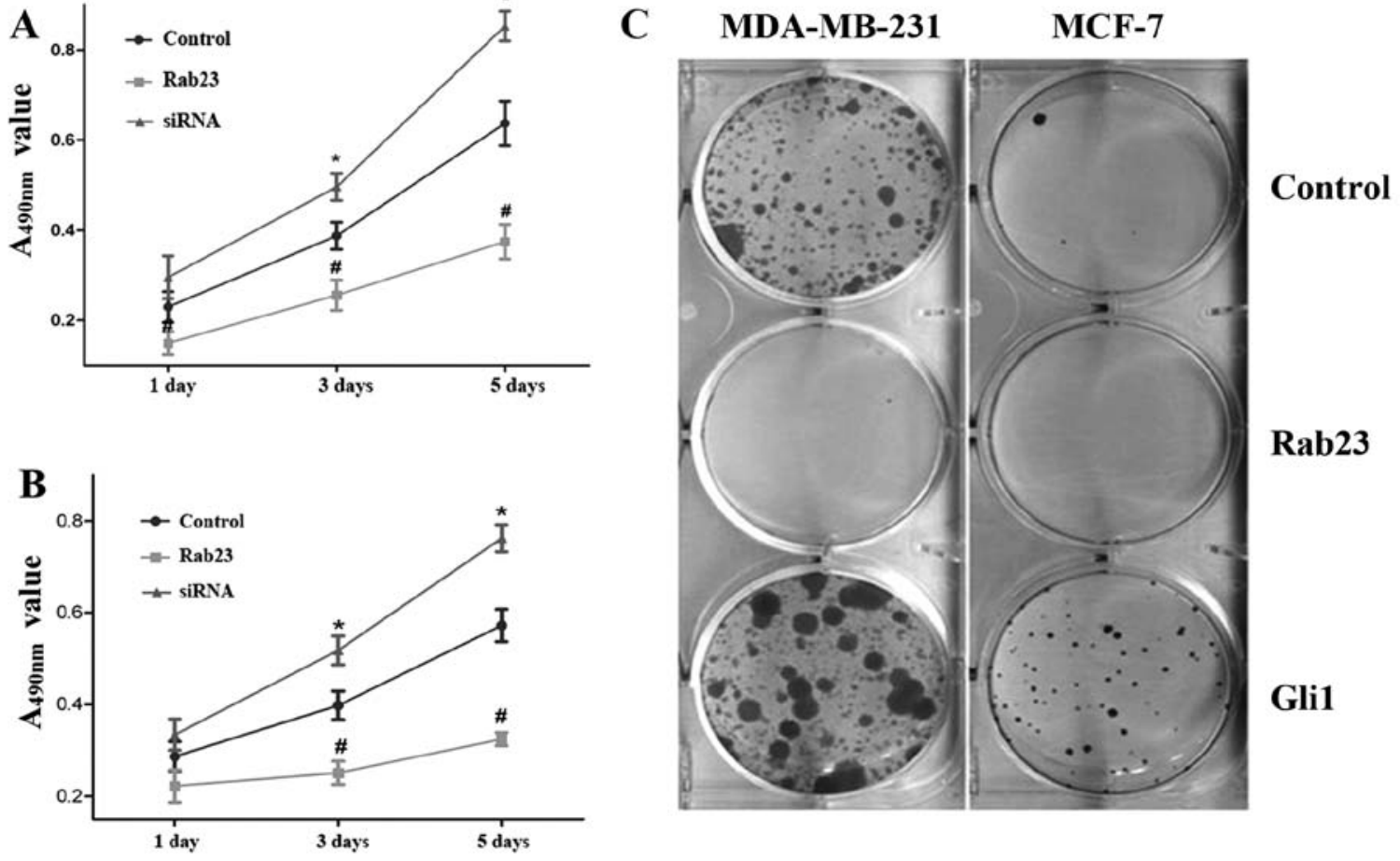

Control

Rab23

Figure 2. Effects of Rab23 on the viability and proliferation of breast cancer cells. Rab23 reduced the cell viability and proliferation rate as determined by the MTT and colony forming assays. (A) MTT assay showed that the absorbance value at $\mathrm{A}_{490 \mathrm{~nm}}$ in the Rab23-transfected group was reduced and the absorbance value in the siRNA-treated group was increased $\left({ }^{*} \mathrm{P}<0.05,{ }^{*} \mathrm{P}<0.05\right)$ compared with the control group in the MDA-MB-231 cells after incubation for 3 and 5 days. (B) MTT assay in the MCF-7 cells ( $\left.{ }^{H} \mathrm{P}<0.05,{ }^{*} \mathrm{P}<0.05\right)$. (C) In the colony forming assay, compared with the control group, the number of colonies formed in the two cell lines transfected with Rab23 plasmids was significantly reduced, and that of the Gli1-expression group was increased.

Rab23 inhibits breast cancer cell viability and proliferation. The cells were transfected with Rab23 siRNA for $48 \mathrm{~h}$. siRNA treatment significantly inhibited Rab23 mRNA expression compared with the control group in the MDA-MB-231 and MCF-7 cell lines. The expression of Rab23 (Rab23/GAPDH ratio) decreased from $0.8453 \pm 0.05252$ to $0.3410 \pm 0.06802$ and from $0.861 \pm 0.05252$ to $0.3821 \pm 0.06802$, respectively, in the MDA-MB-231 and MCF-7 cell lines. Then we used MTT and colony formation assays to detect the effect of Rab23 on breast cancer cell viability and proliferation. MTT assay indicated that the absorbance value at $\mathrm{A}_{490 \mathrm{~nm}}$ in the Rab23-transfected group was reduced compared with the control group $(\mathrm{P}<0.05)$. However, the absorbance value in the siRNA group increased $(\mathrm{P}<0.05)$ (Fig. 2A and B) after incubation for 1, 3 and 5 days. In the colony formation assay, in comparison with the control group, the numbers of colonies formed in the MDA-MB231 and MCF-7 cell lines transfected with Rab23 were significantly reduced, while that of the Gli1-transfected group was increased (Fig. 2C). In the MCF-7 cells, the number of colonies formed was decreased from $18.33 \pm 3.06$ to $4.0 \pm 2.0$ in the Rab23-transfected cells and was increased from $18.33 \pm 3.06$ to $74.33 \pm 9.71$ in the Gli1-transfected cells. In the MDA-MB-231 cells, the number of colonies changed from $124.7 \pm 24.0$ to $7.33 \pm 3.51$ and $124.7 \pm 24.0$ to $242.3 \pm 46.4$, respectively $(\mathrm{P}<0.05)$. These data indicate that Rab23 inhibits the growth of breast cancer cells.

Rab23 inhibits DNA synthesis of breast cancer cells. We detected the effect of Rab23 on the efficiency of DNA synthesis in breast cancer cells by using BrdU labeling.
After transfection of the Rab23 plasmids or siRNA into the MDA-MB-231 (Fig. 3) and MCF-7 (Fig. 4) cells at $40 \mathrm{nmol}$, we found that incorporation efficiency of BrdU in the group transfected with Rab23 was lower than that in the control group $(\mathrm{P}<0.05)$, while the transfection efficacy in the siRNA group was higher $(\mathrm{P}<0.05)$ (Table II). There were no differences between the vector group and the negative control group.

We used FCM to study the effects of Rab23 on the cell cycle distribution of the MCF-7 and MDA-MB-231 cells. The results showed that overexpression of Rab23 protein induced changes in cell cycle progression pattern and caused cell cycle arrest in the G1 phase. The cell proportion in the G1 phase was significantly increased from $58.933 \pm 1.020$ to $68.10 \pm 1.210 \%$ and from $48.525 \pm 2.311$ to $55.00 \pm 0.949 \%$, respectively in the MCF-7 and MDA-MB-231 cell lines, which was accompanied by a decrease in the cell proportion in the $\mathrm{S}$ phase from $29.133 \pm 1.943$ to $22.30 \pm 1.168 \%$ and from $35.125 \pm 1.095$ to $30.175 \pm 0.559 \%$ respectively, compared with the control cells $(\mathrm{P}<0.05)$. Conversely, downregulation of $\mathrm{Rab} 23$ by specific siRNA increased the percentage of cells in the $\mathrm{S}$ phase in both the MCF-7 and MDA-MB-231 cell lines from 29.133 \pm 1.943 to $47.00 \pm 1.000 \%$ and from $35.125 \pm 1.095$ to $50.00 \pm 1.500 \%$ respectively, which was accompanied by a decrease in the cell proportion in the G1 phase compared with the control cells from $58.933 \pm 1.020$ to $47.445 \pm 0.755 \%$ and from $48.525 \pm 2.311$ to $38.00 \pm 0.900 \%$, respectively (Figs. 5 and 6). These results indicate that Rab23 induced arrest in the G1 phase and a decrease in the $\mathrm{S}$ phase population of the cell cycle, and inhibited cell growth and DNA synthesis. Thus, Rab23 significantly reduced DNA synthesis in the breast cancer cells. 


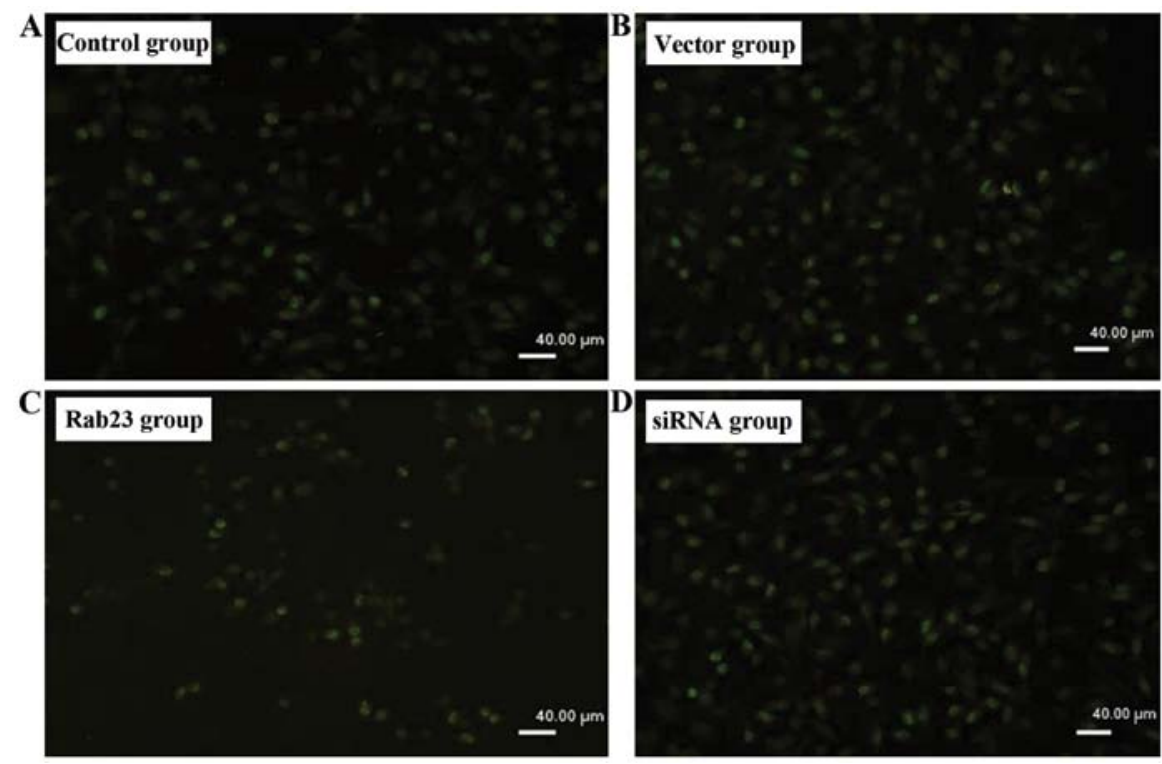

Figure 3. Effects of Rab23 on DNA synthesis in the MDA-MB-231 cells. (A) Control, (B) empty vector, (C) Rab23-expressing and (D) siRNA-treated groups Rab23 reduced the proliferation rate as determined by BrdU labeling. Weaker BrdU labeling was found in the group transfected with Rab23, while that in the siRNA-treated group was higher ( $\mathrm{P}<0.05$ for both groups as per Table II). BrdU, bromodeoxyuridine; siRNA, small interfering RNA.
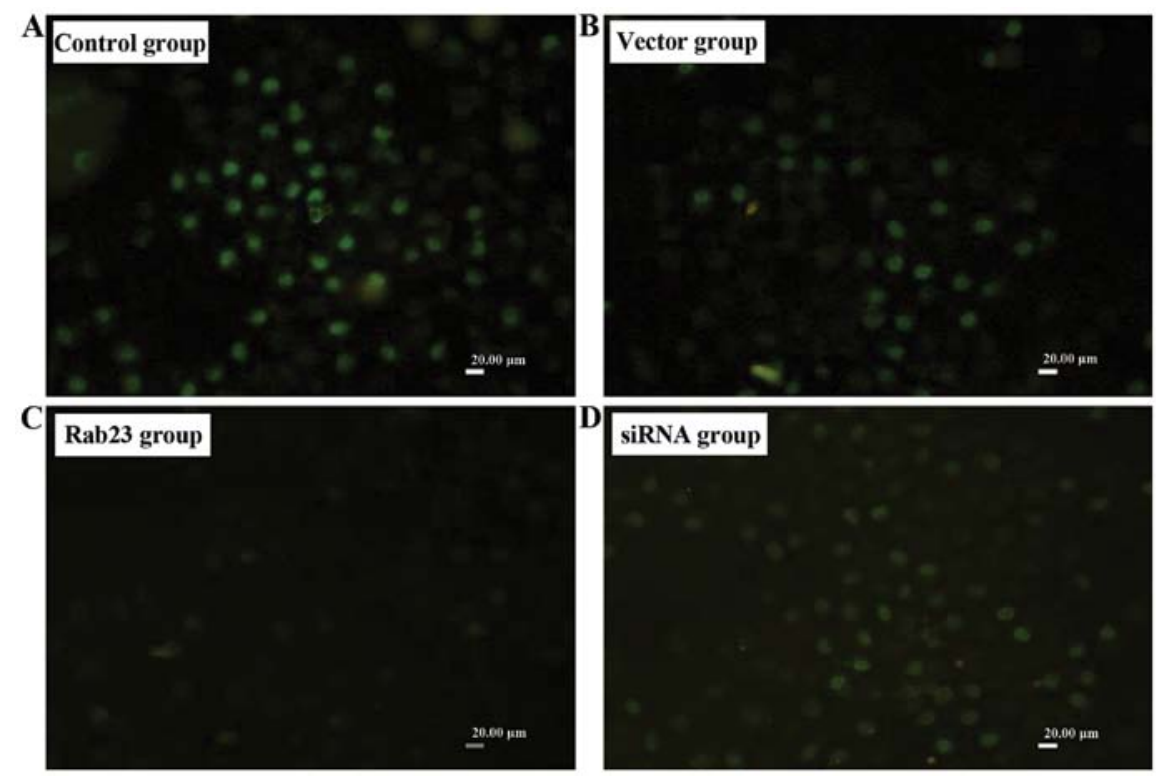

Figure 4. Effects of Rab23 on DNA synthesis in the MCF-7 cells. (A) Control, (B) empty vector, (C) Rab23-expressing and (D) siRNA-treated groups. Rab23 reduced the proliferation rate as determined by the BrdU assay. Weaker BrdU labeling was found in the group transfected with Rab23 than that in the control group, while that in the siRNA-treated group was higher $(\mathrm{P}<0.05$ for both groups as per Table II). BrdU, bromodeoxyuridine; siRNA, small interfering RNA.

Rab23 increases breast cancer cell apoptosis. Following FCM apoptosis analysis, it was observed that Rab23 induced the apoptosis rate of breast cancer cells. After transfection with Rab23, the proportion of cells in the 2 and 4 phases increased when compared with the control and vector groups. The percentage of apoptotic MCF-7 cells increased from $4.633 \pm 1.290$ to $16.70 \pm 1.552 \%$, while the cell proportion of MDA-MB-231 cells increased from $5.867 \pm 1.815$ to $21.50 \pm 2.858 \%$ ( $\mathrm{P}<0.05$ ) (Fig. 7). There was no significant difference between the control and vector group $(\mathrm{P}>0.05)$.

Rab23 downregulates Gli expression. It was found that Rab23 acts as a negative regulator of the Hh signaling pathway, which drives tumorigenesis in several types of human cancers. We found that Gli1 and Gli2 mRNA and protein were expressed in the breast cancer MDA-MB-231 and MCF-7 cells. Following transfection with the Rab23 plasmids, the Gli1 and Gli2 mRNA levels in the MDA-MB-231 and MCF-7 cells were significantly decreased compared with levels in the control group $(\mathrm{P}<0.01)$ (Fig. 8).

\section{Discussion}

Hh signaling is one of the main pathways in embryogenesis which is pivotal to the development of most vertebrate organs and tissues, and its mutation leads to birth defects and the 
Table II. Effect of Rab23 on the incorporation efficiency of BrdU in the breast cancer cells.

\begin{tabular}{lccccc}
\hline & $\mathrm{n}$ & Control & Vector & Rab23 & siRNA \\
\hline MDA-MB-231 & 5 & $32.70 \pm 1.956$ & $30.10 \pm 1.025$ & $20.24 \pm 1.819^{\mathrm{a}}$ & $48.73 \pm 1.555^{\mathrm{a}, \mathrm{b}}$ \\
MCF-7 & 5 & $27.88 \pm 2.178$ & $26.08 \pm 3.047$ & $17.72 \pm 1.767^{\mathrm{a}}$ & $36.20 \pm 2.808^{\mathrm{a}, \mathrm{b}}$ \\
\hline
\end{tabular}

${ }^{\mathrm{a}} \mathrm{P}<0.05$ vs. control; ${ }^{\mathrm{b}} \mathrm{P}<0.01$ vs. Rab23; BrdU, bromodeoxyuridine; siRNA, small interfering RNA.

A

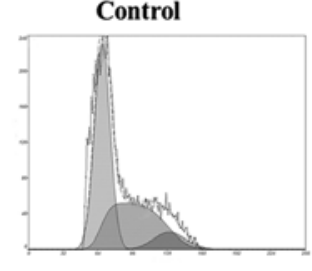

Rab23

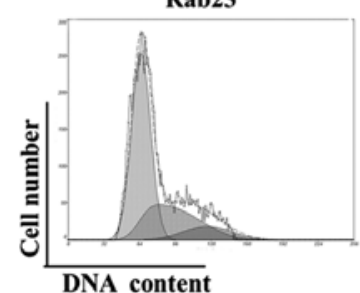

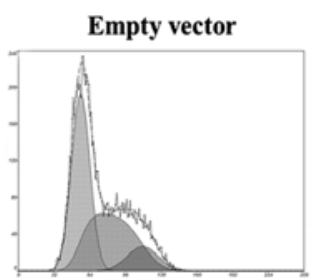

SiRNA

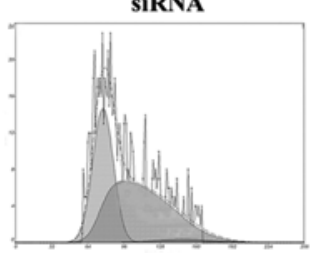

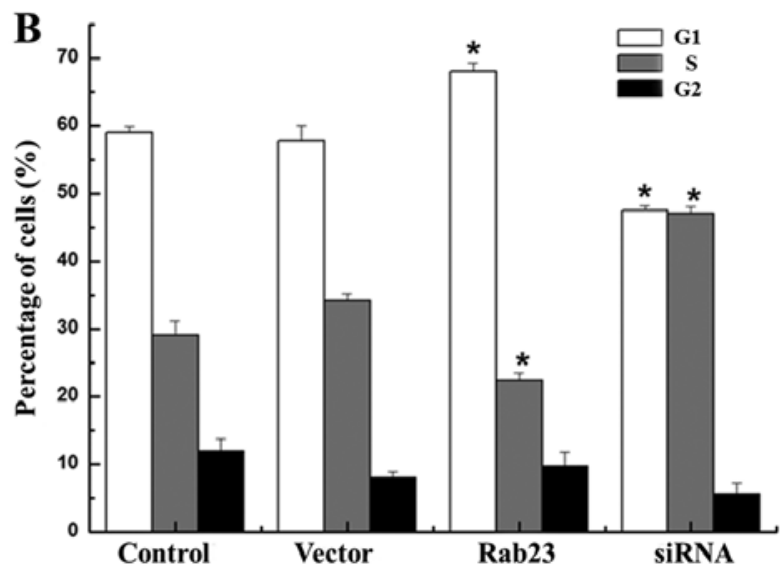

Figure 5. Effect of Rab23 on cell cycle progression in the MCF-7 cells. (A) Expression change in Rab23 altered the cell cycle profile in the MCF-7 cells. (B) The distribution of the cell cycle was analyzed to determine the percentage of cells in each phase of the cell cycle of MCF-7 cells. The data presented are the mean \pm SEM of three independent experiments. White column, G1 phase; gray column, S phase; black column, G2 phase. The results indicate that Rab23 reduced the proportion of cells in the $\mathrm{S}$ phase and increased the proportion of cells in the $\mathrm{G} 1$ phase $\left({ }^{*} \mathrm{P}<0.05\right.$ vs. control).

A
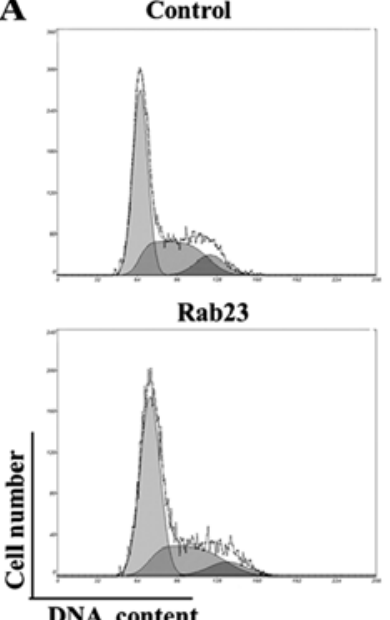

Empty vector

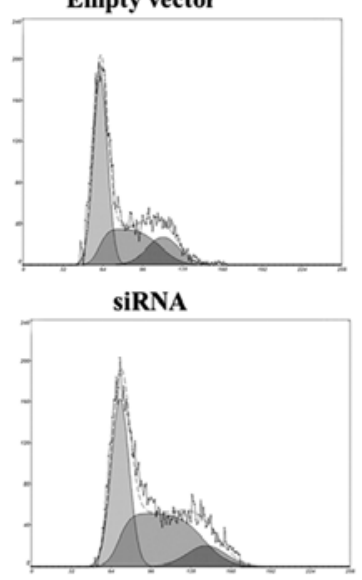

B

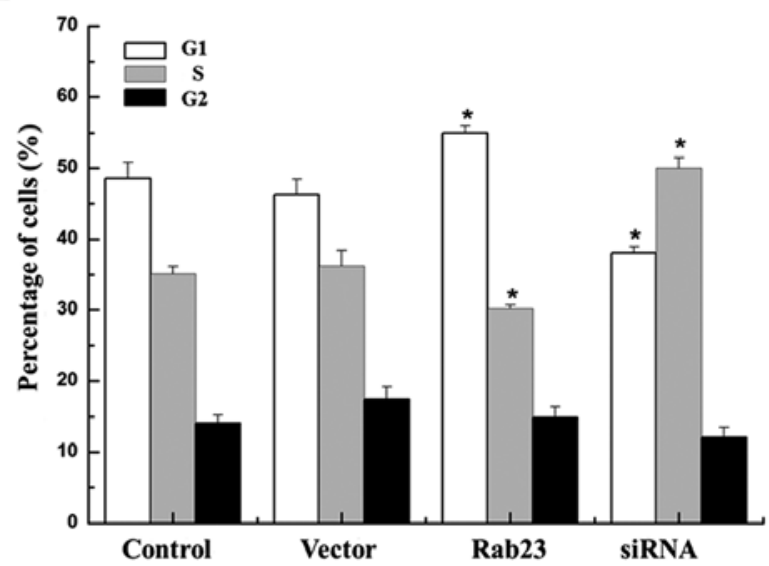

Figure 6. Effect of Rab23 on cell cycle progression in the MDA-MB-231 cells. (A) Expression change in Rab23 altered the cell cycle profile in the MDA-MB-231 cells. (B) The distribution of the cell cycle was analyzed to determine the percentage of cells in each phase of the cell cycle of MDA-MB-231 cells. The data presented are the mean \pm SEM of three independent experiments. White column, G1 phase; gray column, S phase; black column, G2 phase. The results indicate that Rab23 reduced the proportion of cells in the $\mathrm{S}$ phase and increased the proportion of cells in the G1 phase ( $\mathrm{P}<0.05 \mathrm{vs}$. control).

carcinogenesis of numerous types of tumors. Recently, data have shown that abnormal Hh signaling activation is also associated with human tumorigenesis (4). Constitutive activation of the Hh pathway has been found in several types of tumors. Mutations in $\mathrm{Hh}$ pathway components Ptc1 and Smo lead to pathway constitutive activation of tumors in the brain, skin, and muscles, which is considered ligand-independent activation of the Hh pathway $(14,15)$. On the other hand, ligand-dependent pathway activation is important for the growth and survival of a wide variety of cancers, including gastrointestinal tumors, prostate cancer, hematological malignancies and gliomas (16). These data suggest the significance of the Hh pathway as novel diagnostic and therapeutic targets. Further studies have demonstrated that 

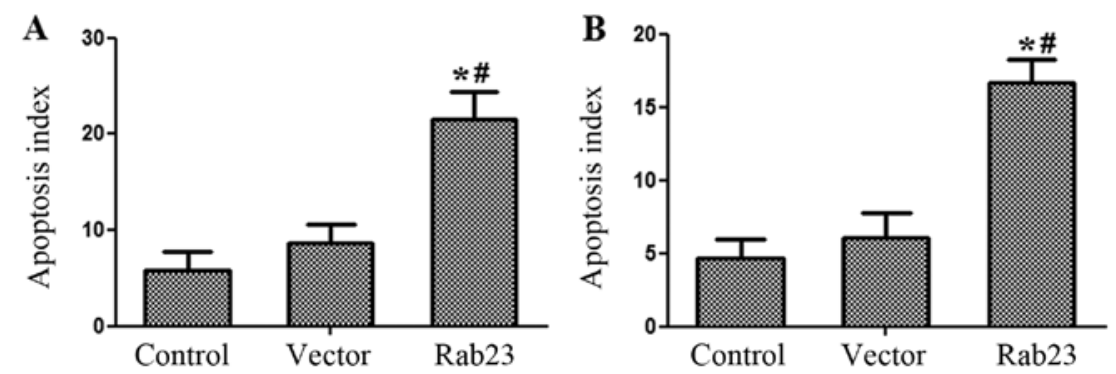

Figure 7. Effect of Rab23 on the apoptosis of breast cancer cells. (A) MDA-MB-231 and (B) MCF-7 cells. After transfection with the Rab23 plasmid in the MDA-MB-231 and MCF-7 cells, the percentage of apoptotic cells was determined by staining with Annexin V and PI followed by FCM. Rab23 increased the apoptosis index of breast cancer cells and induced apoptosis. FCM, flow cytometry; PI, propidium iodide ("P $<0.05$ vs. control, ${ }^{*} \mathrm{P}<0.05$ vs. vector).
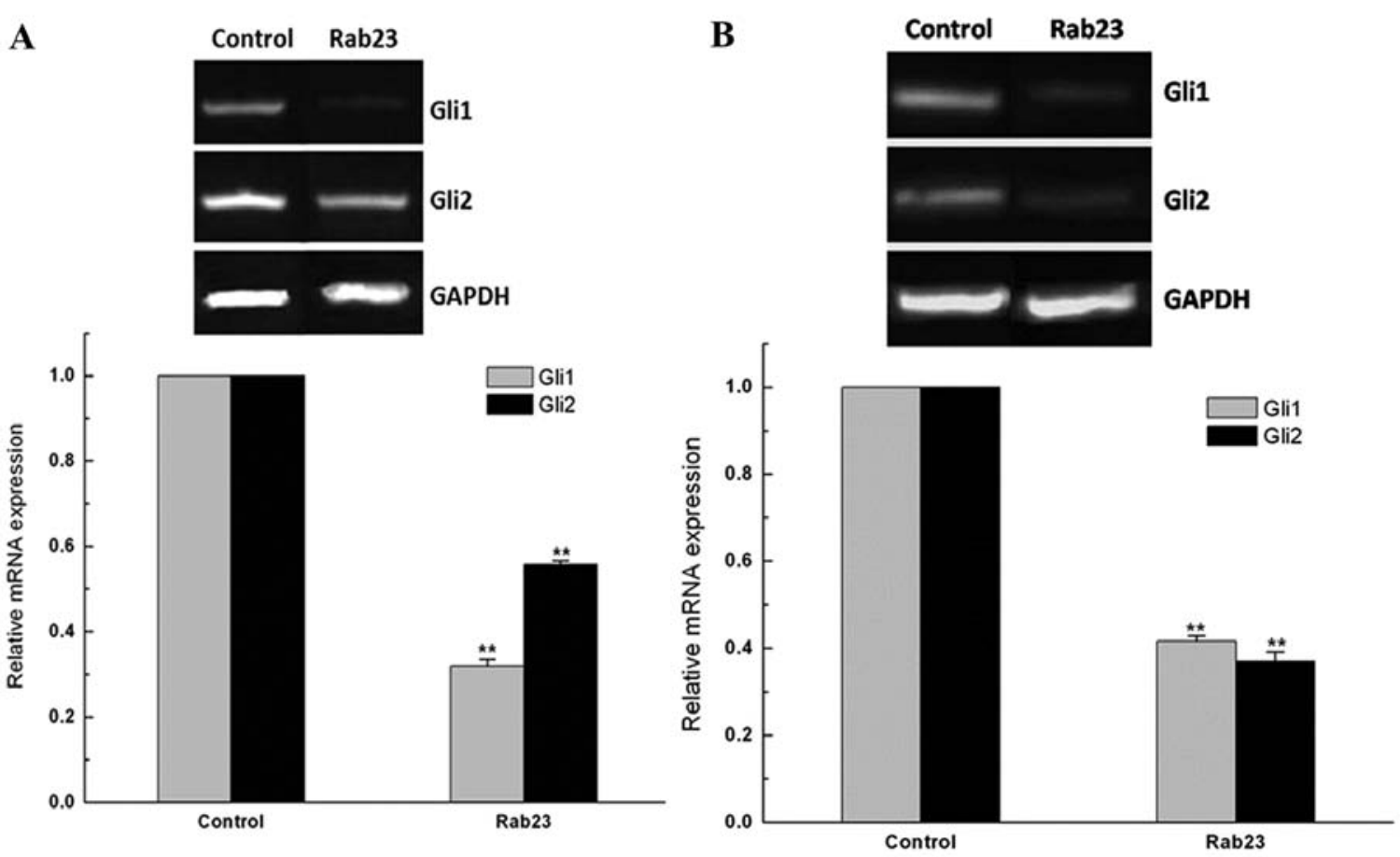

Figure 8. Effects of Rab23 inhibition on Gli1 and Gli2 mRNA expression in breast cancer cells. After transfection with the Rab23 plasmid in the MDA-MB-231 and MCF-7 cells, RNAs were isolated, and gene expression was evaluated by qRT-PCR. (A) MCF-7 and (B) MDA-MB-231 cells. Results are expressed as the mean \pm SEM of three independent experiments. ${ }^{* *} \mathrm{P}<0.01$.

the plant-derived teratogenic steroidal alkaloid cyclopamine, a potent Hh signaling antagonist targeting the SMO protein, suppressed the growth of cancer cells suggesting Hh pathway activation both in vitro and in vivo $(17,18)$. It has been reported that cyclopamine inhibits proliferation and induces apoptosis in various human breast cancer cell lines $(19,20)$. We also found that cyclopamine effectively inhibits the growth and proliferation of breast cancer cells which indicates that $\mathrm{Hh}$ signaling may be abnormally activated in breast cancer. Thus, inhibition of Hh signaling may be a useful method for patients with breast carcinoma (21).

Recently, Rab23 has also been implicated in carcinogenesis. However, the role of Rab23 in tumorigenesis is paradoxical. High Rab23 expression was reported in hepatocellular and gastric cancer patients and was found to be associated with tumor size (22). Another report identified Rab23 as an upregulated gene in nonmalignant diseased gastric tissues compared with normal gastric mucosa (13). In the neural system,
Rab23 may negatively regulate the SHH signaling pathway by controlling the subcellular localization of essential $\mathrm{SHH}$ components $(23,24)$. Since Rab23 is a negative regulator of SHH which can induce malignant carcinoma, we speculated that Rab23 may also contribute to the tumorigenesis in breast cancer.

We first identified whether Rab23 is expressed in three breast cancer cell lines and compared the levels among the cells. We found that Rab23 was expressed at the mRNA and protein levels but had no difference between the MDA-MB-231 and Bcap-37 cells, while there was lower expression in the MCF-7 cells. The expression of Rab23 in the Bcap37 and MDA-MB-231 cells, which are ER-negative, was higher than that in the MCF-7 cells, which are ER-positive, suggesting that Rab23 expression is possibly related with ER expression. This requires further investigation.

A previous study found that Rab23 promotes invasion and its expression is significantly associated with diffuse-type gastric 
cancers (dGCs). This is particularly intriguing since dGCs are phenotypically more invasive than intestinal-type gastric cancers (22). Our results are similar since Rab23 expression was higher in the highly metastatic MDA-MB-231 cells and lower in the low invasive MCF-7 cells. These studies suggest that Rab23 may play a role in tumor metastasis. We propose that the difference in Rab23 expression in breast cancers may be caused by functional changes in the Rab family members. It is known that Ras is a family of genes encoding small GTPases that are involved in cellular signal transduction. It plays an important role in tumorigenesis and tumor metastasis. It may regulate tumor cell growth, differentiation and survival.

In the present study, we observed the effects of Rab23 on the proliferation and apoptosis of breast cancer cells. The results of MTT and in colony forming assays demonstrated that $\mathrm{Rab} 23$ reduced the growth and proliferation of breast cancer cells. Furthermore, the incorporation efficiency of BrdU and FCM showed that Rab23 significantly inhibited cell DNA synthesis. The effect of inhibition was similar to the effects of cyclopamine, a specific signaling inhibitor for the Hh signaling pathway, in breast cancer cells in our previous study (21). Subsequently, FCM apoptosis detection revealed that Rab23 induced the apoptosis of breast cancer cells. These results suggest that the inhibitory effect of Rab23 on breast cancer cells may be due to the inhibition of DNA synthesis and induction of apoptosis in the breast cancer cells.

Rab23, as a new negative regulator of the SHH signaling pathway, has been investigated in many laboratories and it can control the subcellular localization of essential $\mathrm{SHH}$ components. Other studies concerning the effects of Rab23 on carcinomas confirmed that Rab23 plays a role in antagonizing cancer cell survival and increasing the basal rate of apoptosis. The exact cellular mechanism of Rab23 is currently unclear, but data from mouse genetic studies indicate that Rab23 acts downstream of Smo and upstream of Gli proteins. Some genetic studies have indicated that the primary target of Rab23 is the Gli2 activator and that Rab23 and Gli3 repressor have additive effects on mouse neural patterning. We demonstrated that Rab23 suppressed Gli1 transcriptional activity in a $\mathrm{Su}(\mathrm{Fu})$-dependent manner (Suppressor of fused) (25). In order to explore whether the effect of Rab23 on growth, proliferation, and apoptosis of breast cancer cells is regulated through the Hh signaling pathway, we further detected the activated state of the Hh signaling pathway in this study. We found that expression of Gli1 and Gli2 appears to be dependent on Rab23. To date, there are no studies on Gli3 expression in breast cancer cells, and we did not study Gli3 in breast cancer cells. The exact mechanism of Rab23 regulating Hh will require further investigation. The fact that Rab23 expression suppresses Gli1 and Gli2 expression does not rule out the possibility that the effect of Rab23 may be related to the location of Rab23 in the endocytic pathway and the role in facilitating vesicular transport and controlling endocytic progression to lysosomes. In particular, Rab23 expression was higher in the MDA-MB-231 cells, which are more invasive, suggesting that there may be multiple mechanisms under which Rab23 has influence on the proliferation, apoptosis and cell invasion of breast cancer cells.

In conclusion, Rab23 expression was detected in breast cancer cells, and Rab23 represents a potent tumor suppressor in breast cancer, which can inhibit cell growth and proliferation and induced cell apoptosis of breast cancer cells. These effects could be due to the inhibition by Rab23 of Gli1 and Gli2 mRNA expression. The exact molecular mechanism of Rab23 mediation or modulation of the Hh signaling pathway requires additional investigation. Rab23 plays an important role in the tumorigenesis of human breast cancer, and may be a new biological target for the prognosis and treatment of breast cancer. However, further investigations, including detection in clinical samples, are needed to explore and confirm the significance of Rab23 in the pathogenesis and treatment of breast cancer.

\section{Acknowledgements}

This study was supported by grants (no. 31200876 and no. 31371412) from the National Natural Science Foundation of China (NSFC).

\section{References}

1. Benson JR, Jatoi I, Keisch M, Esteva FJ, Makris A and Jordan VC: Early breast cancer. Lancet 373: 1463-1479, 2009.

2. Ingham PW and McMahon AP: Hedgehog signaling in animal development: Paradigms and principles. Genes Dev 15: 3059-3087, 2001.

3. McMahon AP, Ingham PW and Tabin CJ: Developmental roles and clinical significance of hedgehog signaling. Curr Top Dev Biol 53: 1-114, 2003.

4. Saldanha G: The Hedgehog signalling pathway and cancer. J Pathol 193: 427-432, 2001.

5. Kasper M, Jaks V, Fiaschi M and Toftgård R: Hedgehog signalling in breast cancer. Carcinogenesis 30: 903-911, 2009.

6. Naylor TL, Greshock J, Wang Y, Colligon T, Yu QC, Clemmer V, Zaks TZ and Weber BL: High resolution genomic analysis of sporadic breast cancer using array-based comparative genomic hybridization. Breast Cancer Res 7: R1186-R1198, 2005.

7. Nessling M, Richter K, Schwaenen C, Roerig P, Wrobel G, Wessendorf S, Fritz B, Bentz M, Sinn HP, Radlwimmer B, et al: Candidate genes in breast cancer revealed by microarray-based comparative genomic hybridization of archived tissue. Cancer Res 65: 439-447, 2005.

8. Wolf I, Bose S, Desmond JC, Lin BT, Williamson EA, Karlan BY and Koeffler HP: Unmasking of epigenetically silenced genes reveals DNA promoter methylation and reduced expression of PTCH in breast cancer. Breast Cancer Res Treat 105: 139-155, 2007.

9. Mukherjee S, Frolova N, Sadlonova A, Novak Z, Steg A, Page GP, Welch DR, Lobo-Ruppert SM, Ruppert JM, Johnson MR, et al: Hedgehog signaling and response to cyclopamine differ in epithelial and stromal cells in benign breast and breast cancer. Cancer Biol Ther 5: 674-683, 2006.

10. Moraes RC, Zhang X, Harrington N, Fung JY, Wu MF, Hilsenbeck SG, Allred DC and Lewis MT: Constitutive activation of smoothened (SMO) in mammary glands of transgenic mice leads to increased proliferation, altered differentiation and ductal dysplasia. Development 134: 1231-1242, 2007.

11. Iorns E, Turner NC, Elliott R, Syed N, Garrone O, Gasco M, Tutt AN, Crook T, Lord CJ and Ashworth A: Identification of CDK10 as an important determinant of resistance to endocrine therapy for breast cancer. Cancer Cell 13: 91-104, 2008.

12. Liu YJ, Wang Q, Li W, Huang XH, Zhen MC, Huang SH, Chen LZ, Xue L and Zhang HW: Rab23 is a potential biological target for treating hepatocellular carcinoma. World J Gastroenterol 13: 1010-1017, 2007.

13. Kim KR, Oh SY, Park UC, Wang JH, Lee JD, Kweon HJ, Kim SY, Park SH, Choi DK, Kim CG, et al: Gene expression profiling using oligonucleotide microarray in atrophic gastritis and intestinal metaplasia. Korean J Gastroenterol 49: 209-224, 2007 (In Korean).

14. Johnson RL, Rothman AL, Xie J, Goodrich LV, Bare JW, Bonifas JM, Quinn AG, Myers RM, Cox DR, Epstein EH Jr, et al: Human homolog of patched, a candidate gene for the basal cell nevus syndrome. Science 272: 1668-1671, 1996. 
15. Wicking C, Smyth I and Bale A: The hedgehog signalling pathway in tumorigenesis and development. Oncogene 18: 7844-7851, 1999

16. Ruiz i Altaba A, Mas C and Stecca B: The Gli code: An information nexus regulating cell fate, stemness and cancer. Trends Cell Biol 17: 438-447, 2007.

17. Taipale J, Chen JK, Cooper MK, Wang B, Mann RK, Milenkovic L, Scott MP and Beachy PA: Effects of oncogenic mutations in Smoothened and Patched can be reversed by cyclopamine. Nature 406: 1005-1009, 2000.

18. Berman DM, Karhadkar SS, Hallahan AR, Pritchard JI, Eberhart CG, Watkins DN, Chen JK, Cooper MK, Taipale J, Olson JM, et al: Medulloblastoma growth inhibition by hedgehog pathway blockade. Science 297: 1559-1561, 2002.

19. Kubo M, Nakamura M, Tasaki A, Yamanaka N, Nakashima H, Nomura M, Kuroki S and Katano M: Hedgehog signaling pathway is a new therapeutic target for patients with breast cancer. Cancer Res 64: 6071-6074, 2004.

20. Zhang X, Harrington N, Moraes RC, Wu MF, Hilsenbeck SG and Lewis MT: Cyclopamine inhibition of human breast cancer cell growth independent of Smoothened (Smo). Breast Cancer Res Treat 115: 505-521, 2009.
21. Wang HB, Liu YL, Hu YZ and Chi SM: Effect of cyclopamine on growth and proliferation of human mammary carcinoma cell Bcap-37. J Med Postgraduates 20: 567-571, 2007 (In Chinese).

22. Hou Q, Wu YH, Grabsch H, Zhu Y, Leong SH, Ganesan K, Cross D, Tan LK, Tao J, Gopalakrishnan V, et al: Integrative genomics identifies RAB23 as an invasion mediator gene in diffuse-type gastric cancer. Cancer Res 68: 4623-4630, 2008.

23. Evans TM,Simpson F,PartonRG and Wicking C: Characterization of Rab23, a negative regulator of sonic hedgehog signaling. Methods Enzymol 403: 759-777, 2005.

24. Eggenschwiler JT, Bulgakov OV, Qin J, Li T and Anderson KV: Mouse Rab23 regulates hedgehog signaling from smoothened to Gli proteins. Dev Biol 290: 1-12, 2006.

25. Chi S, Xie G, Liu H, Chen K, Zhang X, LiC and Xie J: Rab23 negatively regulates Gli1 transcriptional factor in a $\mathrm{Su}(\mathrm{Fu})$-dependent manner. Cell Signal 24: 1222-1228, 2012. 